\title{
Metagenomic analysis of viral communities in Baikal sponges
}

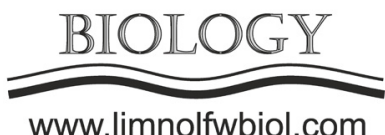

\author{
Butina T.V. ${ }^{1 *}$, Bukin Y.S. ${ }^{1,2}$ \\ ${ }^{1}$ Limnological Institute, Siberian Branch of the Russian Academy of Sciences, Ulan-Batorskaya Str., 3, Irkutsk, 664033, Russia \\ ${ }^{2}$ Irkutsk State University, Faculty of biology and soil studies, Sukhe-Bator Str., 5, Irkutsk, 664011, Russia
}

\begin{abstract}
Sponges are the oldest multicellular invertebrates that represent complex symbioses in aquatic ecosystems. The community of sponges includes various microorganisms: fungi, dinoflagellates, algae, archaea, bacteria, and viruses. The biodiversity and the role of viruses in sponges are poorly studied. The relevance of research in this area is also related to the worldwide problem of sponge diseases. The aim of this study was to elucidate the genetic diversity of viruses in the associated communities of the Baikal endemic sponges Baikalospongia bacillifera and Lubomirskia baikalensis (sick and visually healthy) using the metagenomic approach. We have shown a high taxonomic and functional diversity of DNA viruses in the Baikal sponge communities. Identified sequences belong to the different viral families infecting a wide range of organisms. Viral communities of visually healthy and sick Baikal sponges differed significantly, indicating the changes in the structure of microbial communities of affected sponges.
\end{abstract}

Keywords: metagenomic analysis, virome, viral diversity, viral communities, sponges, Lake Baikal

\section{Introduction}

Sponges are the oldest multicellular invertebrates (phylum Porifera) that represent complex symbioses (holobionts) in aquatic ecosystems. The community of sponges includes various microorganisms: fungi, dinoflagellates, algae, archaea, bacteria, and viruses. Despite the considerable advances in the field of aquatic virology in recent decades, we lack knowledge about viruses of marine and, especially, freshwater invertebrates, including symbiotic organisms. The first studies of the genetic diversity of sponge holobiont viruses demonstrated the high diversity and important role of viruses in the composition of marine sponge communities (Laffy et al., 2016; 2018).

Against the backdrop of global climate change and increasing anthropogenic pressure in Lake Baikal, the mass destruction and death of sponges have been recording since 2011 (Bormotov, 2012). The causes of the disease are still unknown.

The aim of this work was to study the genetic diversity of viruses in the associated communities of the Baikal endemic sponges Baikalospongia bacillifera and Lubomirskia baikalensis (sick and visually healthy) using the metagenomic approach. Sponges B. bacillifera and L. baikalensis are among the most abundant in Lake Baikal.

\section{Materials and methods}

The L. baikalensis sponges were sampled in the Olkhonskiye Vorota Strait, near Maloye More Strait of Lake Baikal. The $B$. bacillifera sponges were sampled in the southern basin of Lake Baikal (near Bolshiye Koty). Pretreatment and preparation of samples for viral analysis was carried out as described previously (Butina et al., 2019; 2020).

The preparation and sequencing of DNA libraries were performed in SB RAS Genomics Core Facility (ICBFM SB RAS, Novosibirsk, Russia) and the Center of Shared Scientific Equipment "Persistence of microorganisms" (ICIS UB RAS, Orenburg, Russia). Sequencing of the libraries was conducted on a MiSeq genome sequencer using MiSeq Reagent Kit v3 (2x300cycles, Illumina). Bioinformatic analysis of data was performed as described previously (Butina et al., 2019; 2020).

\section{Results}

The bulk of the sequences in the datasets was similar to double-stranded DNA (dsDNA) viruses. Bacteriophages of the families Siphoviridae, Myoviridae and Podoviridae had the largest proportion in viromes of $L$. baikalensis and B. bacillifera, which was 


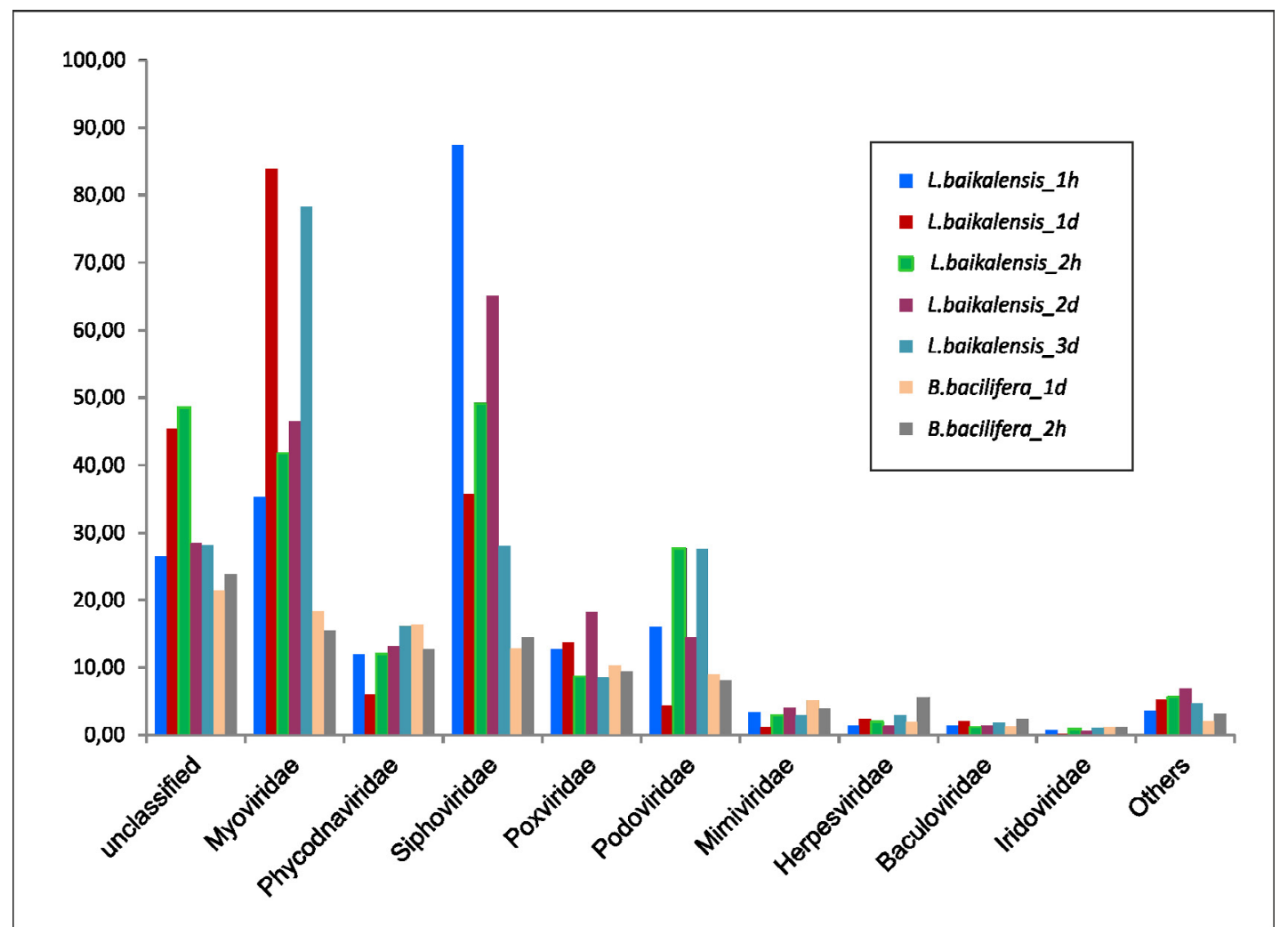

Fig. Percentages of dominant families in sponge viromes. Visually healthy sponges are marked with the letter " $\mathrm{h}$ ", and diseased with the letter "d"

expectable, considering the large abundance of bacteria in the sponge holobionts. The families Phycodnaviridae, Poxviridae, Mimiviridae, Herpesviridae, Baculoviridae, and Iridoviridae were also the most numerous, representing more than $1 \%$ of the sequences (Fig.). Some identified sequences may belong to unknown viruses that infect the sponges.

A comparative analysis revealed that the viral communities of visually healthy and diseased Baikal sponges differed significantly (p-value $<2.2 \mathrm{e}-16$ ); however, no pattern was found in the structure of the communities of healthy or sick specimens. This, probably, indicates the instability and changes in the composition of microbial communities of the affected sponges as well as the propagation of viruses that are not typical of sponges in the normal state.

Thus, the metagenomic analysis of the viral community from the Baikal sponges have revealed for the first time a high genetic, taxonomic and functional diversity of viruses in the associated communities of the $L$. baikalensis and $B$. bacillifera sponges, as well as the differences in viral communities of visually healthy and infected specimens of sponges. In general, our study broadens the understanding of the nature of symbiotic communities in freshwater ecosystems. Future investigations are necessary to elucidate the role of viruses in sponge holobionts, as well as in the mass disease and mortality of these invertebrates.

\section{Acknowledgments}

The study was carried out within the framework of the state task No. 0345-2019-0002 and supported by RFBR and the Government of the Irkutsk Region, project No. 17-44-388080.

\section{References}

Bormotov A.E. 2012. What has happened to Baikal sponges? Science First Hand 32: 20-23.

Butina T.V., Bukin Y.S., Khanaev I.V. et al. 2019. Metagenomic analysis of viral communities in diseased Baikal sponge Lubomirskia baikalensis. Limnology and Freshwater Biology 1: 155-162. DOI: 10.31951/2658-3518-2019-A-1-155

Butina T.V., Khanaev I.V., Kravtsova L.S. et al. 2020. Metavirome datasets from two endemic Baikal sponges Baikalospongia bacillifera. Data in Brief 29: 1-5. DOI: 10.1016/j.dib.2020.105260

Laffy P.W., Wood-Charlson E.M., Turaev D. et al. 2016. HoloVir: a workflow for investigating the diversity and function of viruses in invertebrate holobionts. Frontiers in Microbiology 7. DOI: 10.3389/fmicb.2016.00822

Laffy P.W., Wood-Charlson E.M., Turaev D. et al. 2018. Reef invertebrate viromics: diversity, host specificity and functional capacity. Environmental Microbiology 20: 21252141. DOI: $10.1111 / 1462-2920.14110$ 